\title{
ВОПРОСЫ ПОСТАНОВКИ ЦЕЛИ В УПРАВЛЕНЧЕСКОЙ ДЕЯТЕЛЬНОСТИ ОВД
}

\section{GOAL SETTING QUESTIONS IN THE MANAGEMENT OF THE DEPARTMENT OF INTERNAL AFFAIRS}

\section{A. Rybak \\ R. Danilov}

Summary. Based on the analysis of managerial relations in the internal Affairs bodies, the article concludes that the hierarchy of goals of the elements of the unified management system of the Ministry of internal Affairs of Russia is poorly developed. As a tool for solving this problem, the goal model is proposed, which makes it possible to bring the process of building a hierarchy of goals to a standard basis. The features of the application of the presented model in the field of administrative relations of the Department of internal Affairs make it possible to abandon the use of final quantitative indicators as goals of territorial divisions and move to goals that reflect the level of efficiency of their activities. The technology for calculating the indicator of such a goal is proposed.

Keywords: system, system approach, entropy, organization, problem, task, goal, problem level goal, task level goal, goal model, goal implementation process, unit performance, throughput.
И сследование современной практики управления в органах внутренних дел за последние десять лет позволяет отметить, что основные усилия управленческой структуры в основном направлены на решение краткосрочных проблем, где набор альтернатив решения мал, потребности в многоаспектном анализе невелики, а основные задачи связаны с отслеживанием текущих изменений оперативной обстановки, подготовкой и реализацией решений коллегий различного уровня.

В то же время состояние и структура оперативной обстановки претерпели кардинальные изменения. Прежде всего это связано с нарастающим трендом избыточности многомерной исходной информации о состоянии преступности и ее внешней среды, уменьшением роли рекурсивных и увеличением значения синергетических связей между основными компонентами деятельности ОВД.

Поэтому решение краткосрочных проблем, основанных на изучении только рекурсивных связей не позволя-

\author{
Рыбак Александр Владимирович \\ К.т.н., дочент, Дальневосточный юридический \\ институт МВД России \\ rybak_2908@mail.ru \\ Данилов Роман Михайлович \\ К.т.н., дочент, Дальневосточный юридический \\ институт МВД России \\ danilovroman@mail.ru
}

Аннотация. В статье на основе анализа управленческих отношений в органах внутренних дел делается вывод о слабой проработке вопросов иерархии целей элементов единой системы управления МВД России. В качестве инструмента решения этой задачи предложена модель целей, дающая в03можность привести процесс построения иерархии целей на типовую основу. Особенности применения представленной модели в сфере управленческих отношений ОВД дают возможность отказаться от использования итоговых количественных показателей в качестве целей территориальных подразделений и перейти к целям, отражающим уровень эффективности их деятельности. Предлагается технология расчета показателя такой цели.

Ключевые слова: система, системный подход, энтропия, организованность, проблема, задача, цель, цель уровня проблемы, цель уровня задачи, модель целей, процесс реализации цели, эффективность деятельности подразделения, пропускная способность.

ют решить главную задачу классического управления повышения организованности системы. Статистические данные по оперативной обстановке за последнее десятилетие подтверждают наличие этой проблемы.

С одной стороны, за годы, прошедшие после реформы ОВД достаточно существвенно улучшился имидж органов внутренних дел, принесла положительные результаты борьба с коррупцией среди сотрудников и работников органов внутренних дел. За период с 2006 г. по 2018 г. эти положительные результаты сопровождаются стабильным ежегодным уменьшением количества зарегистрированных преступлений в целом по стране.

В то же время в управленческой деятельности территориальных подразделений сформировались ряд негативных явлений, которые требуют к себе особого внимания.

Прежде всего обращает на себя внимание достаточно острый некомплект сотрудников по наиболее важ- 
ным направлениям деятельности территориальных подразделений: следствия, дознания, уголовного розыска, участковых уполномоченных. Дополнительно к этому наблюдается высокий уровень ротации по этим направлениям. Как следствие - уменьшение среднего стажа работы и снижение профессионализма сотрудников.

В то же время структура преступности претерпела в последнее время значительные изменения. Наметился ежегодный трендувеличения тяжких и особо тяжких преступлений при уменьшении количества грабежей и ряда других насильственных преступлений. На этом фоне быстрыми темпами увеличивается количество сложных преступлений, таких как мошенничество, особенно связанное с использованием информационно-телекоммуникационных технологий или в сфере компьютерной информации. В целом, массив таких преступлений за последние 5 лет увеличился более чем в шесть раз с 43,8 тыс. преступлений в 2015 году до 294,4 тыс. в 2019 году. По итогам первого полугодия 2020 г. их количество возросло почти на 92\% (до 225,5 тыс.) [1]. Более того, в 2019 году общее количество преступлений, зарегистрированных на территории России, увеличилось.

Таким образом в соответствии с новыми трендами в преступности повышаются требования к профессиональной подготовке руководителей и сотрудников территориальных подразделений. Но системных решений в практике управленческих отношений в этом направлении не просматривается.

Кадровая проблема усугубляется тем, что в современном российском обществе стало все трудней находить достойный кадровый резерв в виде «скамейки запасных» для решения подобного рода задач. Молодые люди, которые служат в органах внутренних дел или которые по возрасту, по образованию и по уровню интеллектуальных способностей потенциально могут прийти на службу в органы внутренних дел, обладают набором жизненных ценностей, которые несколько противоречат требованиям, предъявляемыми к сотруднику органов внутренних дел. Невысокий уровень денежного довольствия по сравнению с уровнем психической и физической нагрузки, ненормированный рабочий день, необходимость отказа от ряда конституционных прав, явно завышенные требования к здоровью - это далеко не полный перечень факторов, которые негативно влияют на выбор профессии сотрудника органов внутренних дел.

То есть сложилось явное противоречие между требованиями, которые предъявляются к профессиональным качествам сотрудников в настоящее время и сложившейся технологией отбора, воспитания и мотивации перспективных молодых людей для службы в системе
МВД России. Снятие этого противоречия лежит за пределами решения отдельных вопросов управления (увеличение денежного довольствия, премиальные выплаты и пр.) и требует системного подхода.

Под системным подходом понимается методология исследования любых процессов, явлений, объектов и проблем как систем (при условии, что таковыми их можно представить).

Под системой принято понимать идеальную или материальную целостность, состоящую из взаимосвязанных элементов, объединенных единой целью. Следовательно, единство цели их функционирования и развития является основным системообразующим фактором.

Таким образом, чтобы представить структуру МВД России как систему управления и оценить степень ее организованности необходимо выстроить иерархию целей составляющих ее элементов: центрального аппарата, территориальных органов и организаций системы МВД. Решение этого вопроса представляет на сегодняшний день довольно сложную задачу, так как нормативная база системы МВД содержит лишь формулировки задач, направлений и функций подразделений, но не содержат целей. Поэтому вопрос иерархии целей элементов единой системы управления МВД России остается открытым и требует к себе в настоящее время более глубокого изучения.

Содержанием понятия цель, ее активностью или пассивностью по отношению к объекту управления всегда занимались, занимаются на различных научных направлениях: формальном, логическом, философском и т.п. В современном информационном обществе, когда отсутствует дефицит информации, но имеет место «проклятье многомерности» ее оценки эти вопросы требуют особой проработки. Без построения иерархии целей сложно принять решение, что выбрать в качестве цели - итоговый количественный показатель деятельности подразделения (например, количество административных протоколов, которые необходимо составить за единицу времени) или минимизацию времени, при выполнении поставленной задачи.

Чтобы разобраться с этой многомерностью, рассмотрим цель, когда она обуславливает необходимость какой-то деятельности, которая может быть выражена вопросами, требующими решения.

Вопросы, встречающиеся в процессе управления социальными системами и органами внутренних дел, в частности, столь разнообразны по своему характеру, по масштабу и по длительности что, прежде всего, их необходимо сгруппировать на основе единого критерия. 


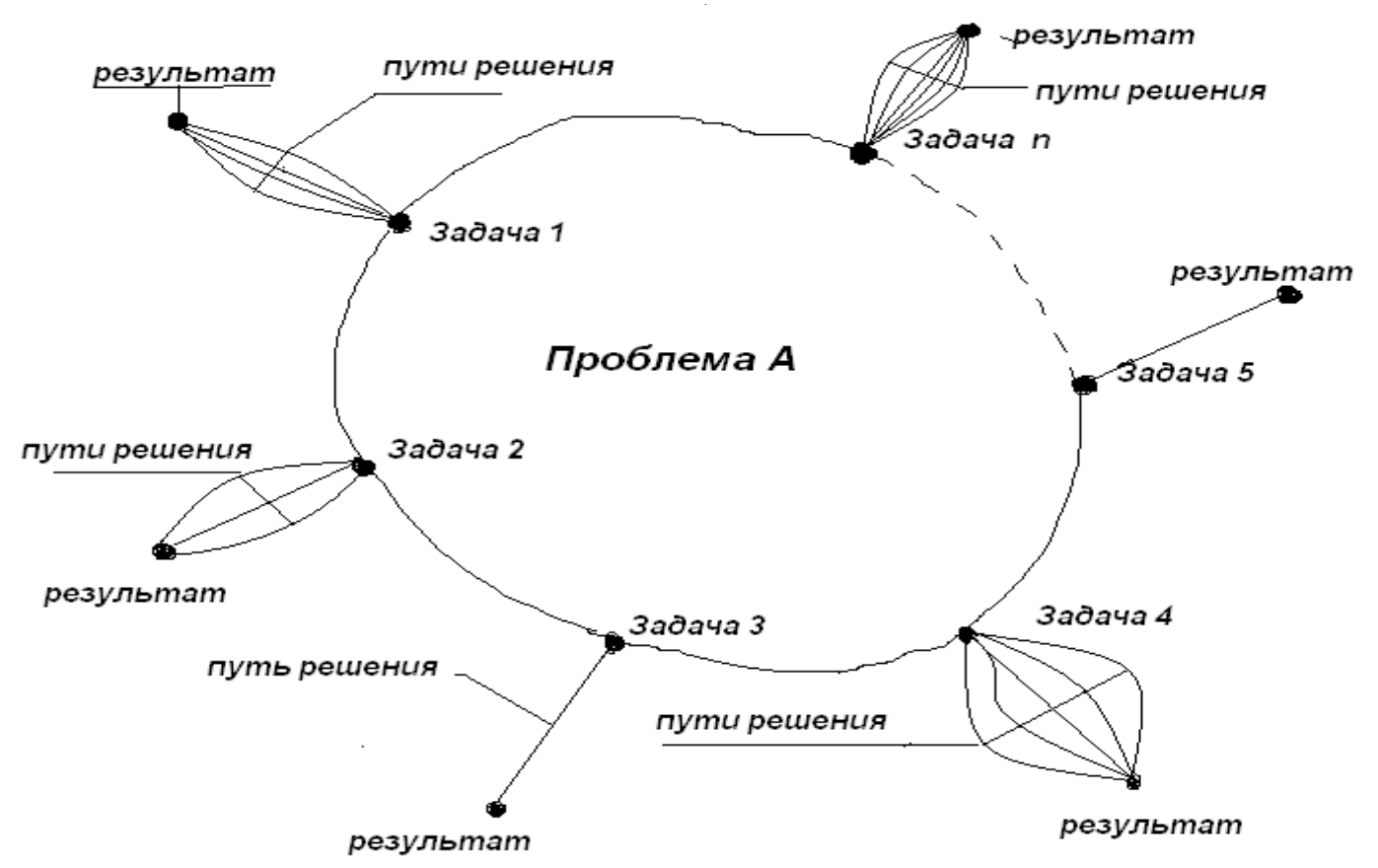

Рис. 1. Соотношение проблемы и задачи

Таким достаточным критерием можно выбрать величину неопределенности в вопросе, для устранения которой либо есть пути решения, либо их нет в рассматриваемый момент времени.

Отсюда появляются два принципиально различных вида вопросов, которые условно можно разделить на проблемы и задачи [3].

Под проблемой будем понимать разновидность вопроса, имеющего четко поставленную цель, но пути достижения которой не могут быть установлены достаточно строго в данный момент времени (рис. 1).

Под задачей будем понимать разновидность вопроса, имеющего четко поставленную цель и конкретный путь (несколько путей) ее решения.

Проблема А может быть решена, или предполагается ее решение с помощью нескольких задач (задачи 1, 2 и т.д.). Число путей решения проблемы, как это следует из данного нами выше определения, не может быть выявлено достаточно четко в рассматриваемый момент времени Т. Для задач, как это видно на рис. 1, может быть либо один путь решения (например, для задач 3, 5), либо несколько путей решения (задачи 1, 2, 4, N) и т.д.

Если для задач цели могут быть определены предельно четко и ясно выражены конкретными количественными показателями, то цели проблемы можно только приблизительно оценить на уровне качественных или количественных показателей, но с большим разбросом. На основании типов вопросов, требующих решения, формулируются и два вида целей.

Цель уровня проблемы - объективная социальная необходимость, которая может быть установлена и выражена набором количественных или качественных показателей, состав которых и пределы отклонений не могут быть определены в полной мере. Например, это может быть повышение эффективности работы органов внутренних дел в целом по стране.

Цель уровня задачи - объективная социальная необходимость, которая может быть установлена и выражена набором количественных показателей с заданными пределами отклонений.

Например, это может быть повышение эффективности работы следственных подразделений территориальных органов на основе внедрения новых критериев оценки следственной работы, связанных с учетом ее организованности.

Отсюда появляется возможность представить цели в виде модели, два вида целей, процесс их реализации с оценкой его эффективности [5] (рис. 2).

Цель 1 условно будем называть целью-потребностью. Этот тип цели в основном формируется на уровне 


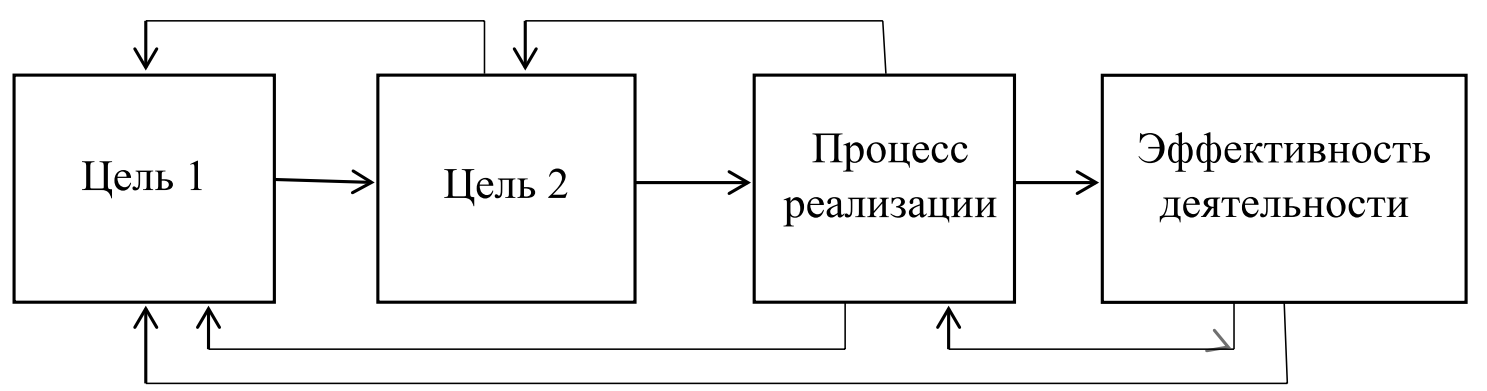

Рис. 2. Модель целей

центрального аппарата МВД России. Формулирование цели на этом уровне управления связано с необходимостью учета и формализации, наряду с показателями работы органов внутренних дел, большого массива криминологических факторов социального, экономического, политического, психологического и пр. характера. Это привносит в цель-потребность высокую долю неопределенности. По этой причине, как уже было сказано выше, формализация такой цели представляет чрезвычайно сложный процесс, что ограничивает возможность применения на этом уровне итоговых количественных показателей, задающих стратегию и тактику работы органов внутренних дел. Здесь возникает необходимость рассмотрения в качестве дополнительной цели заданного уровеня эффективности деятельности территориальных подразделений, оценивающего количественно степень их организованности при выполнении поставленных задач в сложившейся оперативной обстановке. Подробно расчет уровня эффективности будет рассмотрен ниже.

Цель 2 (уровень задач) непосредственно связана с процессом реализации, ее можно назвать технологической, т.е. такой целью, которая наряду с ее формальным представлением может служить основой для сравнения целевых функций процессов различного характера как по масштабу, так и по содержанию. Очевидно, что такое сравнение необходимо для выявления уровня решаемых задач, а также для интеграции задач по их содержательным характеристикам, что позволит совместно рассматривать задачи, решаемые по различным видам правоохранительной деятельности, общих по целевой функции: работа дежурных частей, следственных подразделений, уголовного розыска, участковых уполномоченных. Таким образом, этот тип цели наиболее корректно формируется на уровне территориальных подразделений.

Под процеессом реализац̧ии понимается любой вид целенаправленной деятельности, включающей все необходимые аспекты для достижения поставленной цели.

Процессами могут быть процесс оперативно-розыскной деятельности, процесс расследования преступлений, процесс охраны общественного порядка и пр.
При этом в процессе реализации цели 2 (уровня задачи) имеют место следующие основные составляющие, которые сами по себе также являются процессами:

1. Процесс разработки нормативной и методической документации, определяющей процессы реализации целенаправленной деятельности.

2. Собственно процесс деятельности подразделения на основе разработанной технологии для решения поставленных задач.

3. Процесс обеспечения, который включает все необходимые элементы обеспечения: кадровое, материальное, финансовое, психологическое и пр.

4. Процесс управления. Задачей этого процесса является координация трех вышеназванных процессов для достижения общей цели с использованием минимальных ресурсов. Процесс управления включает краткосрочное прогнозирование, долгосрочное и краткосрочное планирование, оперативное

4. Процесс развития включает в себя технологию решения перспективных задач подразделения. Процесс развития должен обеспечить перспективу совершенствования организации деятельности подразделения при изменении оперативной обстановки.

Таким образом, технологическая цель может быть представлена в виде дерева технологических целей процессов: организация деятельности; обеспечение; управление; развитие.

В связи с тем, что эти процессы выполняют различные функции и характеры направленности их действий отличны друг от друга, появляется необходимость типизировать число уровней и состав структурных элементов в глубину каждого процесса, так как только последнее может обосновать выявление прямых и обратных связей на всех этапах получения конечного результата: от идеи до реализации.

Если, например, технологическая цель получения конечного результата составлена таким образом, что сте- 
пень детализации соответствует минимальным автономным составляющим системы (например, следственному отделу районного отдела полиции), а цель обеспечения включает только крупные составляющие системы (уровня УМВД РФ по региону), то имеет место разный масштаб представления указанных видов технологических целей. В этом случае объединение связями данных видов целей практически не может привести не только к ожидаемому результату, но даже |к построению строгой системы управления, включающей координацию работ по разномасштабным элементам. Решение о степени детализации технологических целей должно приниматься на уровне центрального аппарата.

Блок «Эффективность деятельности» определяет степень организованности системы управления подразделением при решении служебных задач. В качестве синонима понятия организованности можно использовать понятие порядка. Отсюда задачей управления их деятельностью является уменьшения беспорядка. Количественной мерой измерения беспорядка в системе является ее особая характеристика - энтропия. Она характеризует соотношение организованности и дезорганизованности в системе, предопределяет поведение людей, в данном случае сотрудников, в сложной оперативной обстановке. Степень организованности системы тем выше, чем меньше количество содержащейся в ней энтропии. Но здесь нельзя с точки зрения управления абсолютизировать значение организованности в системе. Стремление уменьшить энтропию до нуля приводит к стагнации системы, уменьшению креативности во взаимодействии ее элементов. При этом энтропия системы вытесняется во внешнюю среду, что формирует между ними противоречие, которое при достижении определенного уровня может привести к разрушению системы. В части, касаемо управления территориальными подразделениями, это выглядит как потеря мотивации сотрудников к выполнению своих служебных обязанностей и профессиональному росту, стремление найти другую область применения своих сил и способностей вне деятельности правоохранительных органов. Именно в этом причина затяжного некомплекта по основным направлениям деятельности территориальных подразделений: следствие, дознание, уголовный розыск, деятельность участковых уполномоченных.

Таким образом задачей эффективного управления является поддержание определенного баланса между организованностью и беспорядком при безусловном доминировании организованности.

Здесь возникает вопрос - как определить этот баланс, как рассчитать границу между организованностью и неорганизованностью в виде количественного параметра, определив его границей эффективного и неэф- фективного управления? Активной целью управления подразделением в данном случае может быть достижение этого параметра, либо его превышение на заданный диапазон.

Технология расчета этого параметра основана на использовании модели динамического равновесия четырех показателей оперативной обстановки [4]:

$\alpha, \gamma, R, V$. Исходя из того, что форма зависимости между используемыми параметрами, не линейная, а экспоненциальная, модель представлена в следующем виде:

$$
\alpha=\frac{\gamma \cdot R}{V} \cdot\left(1-e^{-\frac{V}{\gamma \cdot R}}\right)
$$

где $\alpha$ - рассматривается, как показатель границы эффективного управления;

$\gamma$ - показатель эффективной (в зависимости от поставленной задачи) «пропускной способности» одного сотрудника за отчетный период;

$R$ - число сотрудников территориального подразделения;

$V$ - реальная нагрузка на подразделение в виде количества объектов, отработанных подразделением за отчетный период (например, количество уголовных дел в производстве у следственного подразделения по данным статистики).

Определяющим параметром применения этой модели для поставленной задачи является $\gamma$ - величина, определяющая «пропускную способность» одного сотрудника за отчетный период. Представим в краткой форме технологию ее расчета, например, для следственных подразделений [2].

1. Все преступления, регистрируемые на территории подразделения, предварительно методом экспертных оценок классифицируются по степени сложности и трудоемкости расследования на отдельные категории (например, на 8 категорий).

2. Методом экспертных оценок для каждой категории определяется количество преступлений, которое может расследовать на подведомственной территории один следователь за один год. Представим эти показатели в виде числовой последовательности $k_{1}, k_{2}, k_{3}, \ldots, k_{8}$.

3. На основе данных статистической отчетности определяем долю каждой категории преступлений в общем количестве преступлений, зарегистрированных на территории подразделения в отчетный период. Представим ее также в виде числовой последовательности $d_{1}, d_{2}, d_{3}, \ldots, d_{8}$. 
4. Рассчитываем $\gamma$ - «пропускную способность» одного сотрудника методом расчета средневзвешенной величины.

$$
\begin{aligned}
& \gamma=k_{1} * d_{1}+k_{2} * d_{2}+k_{3} * d_{3}+k_{4} * d_{4}+k_{5} * d_{5}+k_{6} * d_{6}+ \\
& +k_{7} * d_{7}+k_{8} * d_{8} \ldots
\end{aligned}
$$

5. В полученной величине «пропускной способности» $\gamma$ в соответствии с логикой ее расчета учтена не только сложившаяся в отчетный период структура преступности и трудоемкость расследования зарегистрированных преступлений на территории подразделения, но и требуемый уровень профессиональной компетентности ее сотрудников. На этом основании, подставив полученное значение $\gamma$ в формулу (1), получаем возможность определить нижнюю границу эффективности деятельности подразделения. В соответствии с этой формулой увеличение количества зарегистрированных преступлений $V$ или увеличение сложности и трудоемкости расследования преступлений за отчетный период (учтено в $\gamma$ ) ведет к уменьшению значения границы эффективности работы подразделения. Обратный процесс ведет к увеличению значения этой границы.

Следовательно, определив в качестве технологической цели уровень достаточной эффективности деятельности подразделения, мы получаем возможность избавиться от влияния внешней среды (структурной и количественной динамики преступности) на достижение поставленной цели. При этом уровень эффективности деятельности подразделения, его степень организованности подвергается непосредственному измерению.

Таким образом, подводя итог сказанному, модель, представленная на рис. 2, позволяет принципиально не считать цель уровня проблемы или цель уровня за- дачи единственно активными элементом модели. Ими может быть любой из элементов модели, например процесс реализации. Действительно, можно сформулировать много целей, но осуществить их практически невозможно из-за отсутствия технологии процесса реализации, которая сама по себе может являться проблемой.

Проблемой может быть и эффективность деятельности подразделения, даже если мы знаем необходимые ее показатели. Если же на практике нельзя реализовать технологию достижения такой эффективности, то результаты деятельности подразделения будут иметь большой разброс, как со стороны состава самих показателей, так и со стороны их отклонений. Следовательно, все элементы модели (рис. 2) можно условно считать и активными, и пассивными.

Активность или пассивность элементов модели определяется только в процессе построения дерева целей, при наличии конкретно поставленной задачи.

Если при построении дерева целей окажется, что хотя бы один из элементов, например процесс реализации, не может быть реализован в рассматриваемый момент времени, а элемент «цель» явился инициатором построения дерева целей, то цель в этом случае - активный элемент. Наоборот, если дерево целей строится исходя из оптимизации ресурсов, а именно, возможностей получения требуемой эффективности и процесса ее реализации, то цель в этом случае является пассивным элементом.

Таким образом, представленная модель целей позволяет выработать типовую технологию построения иерархии целей, начиная от выявления проблемы и формулирования целей-потребностей до расчета количественного показателя активного элемента технологической цели.

\section{ЛИТЕРАТУРА}

1. Состояние преступности в России за январь — июнь 2020 года. Статистический сборник. - Москва. Генеральная прокуратура Российской Федерации. 2020. (Сборник подготовлен на основании формы федерального статистического наблюдения № 4-ЕГС и ведомственного отчета МВД России формы 1-А).

2. Рыбак А. В. Оценка эффективности деятельности следственных подразделений на основе применения методов системной динамики // Научный портал МВД России. 2016. № 3 (35). С. 99-103.

3. Рыбак А. В. Системный анализ в диссертационном исследовании //Технология и особенности применения в сфере юридических наук. Научный журнал.2018. № 6. С. 231-326.

4. Минаев В. А. Кадровые ресурсы органов внутренних дел: современные подходы к управлению. Монография.— М.: Академия МВД СССР, 1991.

5. Стабин И.П, Моисеева В.С. Автоматизированный системный анализ. - М.: Машиностроение, 1984. 